\title{
UTILIZAÇÃO DE BIOMARCADORES EM PEIXES COMO FERRAMENTA PARA AVALIAÇÃO DE CONTAMINAÇÃO AMBIENTAL POR MERCÚRIO (Hg)
}

\author{
Taíse Bomfim de Jesus ${ }^{1,2^{*}}$ \& Carlos Eduardo Veiga de Carvalho ${ }^{1}$ \\ ${ }^{1}$ Laboratório de Ciências Ambientais,Universidade Estadual do Norte Fluminense Av. Alberto Lamego, 2000, Parque Califórnia, 28015620, Campos \\ dos Goytacazes-RJ,Tel: 22-27261403 Fax:22-27261472. \\ ${ }_{2}^{2}$ Programa de Pós-Graduação em Ecologia e Recursos Naturais, Centro de Biociência e Biotecnologia, UENF, Av. Alberto Lamego, 2000, Parque \\ Califórnia, CEP:28015620, Campos dos Goytacazes-RJ. \\ *E-mail: taisebj@hotmail.com
}

\section{RESUMO}

O estudo dos biomarcadores é uma ferramenta de avaliação do efeito tóxico de substâncias poluentes em ecossistemas aquáticos, que através do desenvolvimento e aplicação de técnicas de exposição ou efeito em três níveis de complexidade (individual, celular e molecular), tornando possível a elucidação da relação causaefeito e dose-efeito na avaliação de risco à saúde. O mercúrio e seus compostos são considerados importantes poluentes ambientais e são quimicamente ativos, e, dependendo da concentração atingida nos tecidos animais, podem desnaturar proteínas e inativar enzimas e alterar a atividade celular. No monitoramento aquático, os peixes funcionam como bons indicadores de poluição, e o grau de eficiência de cada biomarcador depende do organismo (tamanho, sexo e idade) e da posição deste na cadeia trófica, por isso, estudos de qualidade ambiental que utilizam os mais diversos tipos de biomarcadores refletem com melhor precisão os efeitos do possível contaminante no organismo.

Palavras-chave: Ecossistema aquático, indicadores de poluição, efeito tóxico, monitoramento.

\begin{abstract}
USING BIOMARKERS IN FISH TO DETECT ENVIRONMENTAL CONTAMINATION BY

MERCURY. Biomarkers can be used to evaluate the toxic effects of pollutants to aquatic environments. Through studying the effects of exposure to pollutants in three levels of complexity - individual; cellular and molecular - the actual health risk related to causes/ doses/ effects can be evaluated. Mercury and its derivates are considered important environmental pollutants that are highly reactive, which upon certain concentrations within tissues can denature proteins and inactivate enzymes, thus impairing cellular activities. Fish can be suitably coupled with biomarkers to serve as indicators of pollution in aquatic environments. The efficiency degree of each biomarker will depend of the fish used (e.g. its size, sex, and age) and on its trophic position in the system. This way, environmental quality studies that employ a wide range of biomarkers more are capable of returning a precise evaluation of the possible effects of a contaminant on a given organism.
\end{abstract}

Keywords: Aquatic ecosystems, pollution assessement, indicators of pollution, toxic effects

\section{INTRODUÇÃO}

O crescimento populacional verificado nas últimas décadas, acoplado ao aumento do avanço tecnológico e ao aumento na geração de produtos industriais, inclusive a manufatura de produtos químicos como fertilizantes, inseticidas e herbicidas, tem levado a economia global a uma expansão do nível de compostos xenobióticos no ecossistema aquático. Tal fato vem contribuindo para a redução da qualidade dos diversos compartimentos ambientais (água, sedimento, ar e solo), bem como para o comprometimento da saúde dos seres vivos que habitam esses ecossistemas (Cajaraville et al. 2000).

Através das primeiras discussões científicas mundiais sobre prevenção de impactos ambientais, em 1992, a USEPA lançou uma nova abordagem, intitulada "Avaliação de risco". Esta abordagem incorpora informações derivadas cientificamente, com preocupações sociais e econômicas, para projetar as conseqüências da ação de estressores de origem antrópica sobre o ambiente e, com base nesse modelo projetado, poderem definir ações para evitá-las.

A exposição constante da biota aquática a subs- 
tâncias tóxicas lançadas no meio ambiente, como mercúrio e suas espécies químicas, é capaz de interagir com o organismo vivo causando múltiplas alterações que podem gerar graves conseqüências em populações, comunidades ou ecossistemas, dependendo do grau de contaminação e do tempo de exposição, como pode ser observado na Figura 1.

Para avaliar o efeito nocivo de um contaminante lançado no ambiente quando este está abaixo do limite legislado, utilizam-se testes de toxicidade, analisados por estimativa de pontos, normalmente expressos por concentrações letais, efetivas ou inibidoras que causam, respectivamente, mortalidade, anormalidade ou inibição a $50 \%$ dos organismos expostos. Entretanto, Denton et al. (1994) provaram, com base em uma série de dados, que o valor aproximado, a partir do qual o efeito começa, está sempre abaixo de $25 \%$, o que torna a avaliação de riscos com base em valores de $50 \%$, uma temeridade (Nascimento et al. 2006). A impossibilidade de confiança dos dados de toxicidade, ainda utilizados hoje, torna o uso dos biomarcadores mais promissores para a detecção preventiva de efeitos adversos.

Biomarcadores são hoje definidos como respostas biológicas adaptativas a estressores, evidenciadas como alterações bioquímicas, celulares, histológicas, fisiológicas ou comportamentais (Depledge 1992, Depledge 1993). Em 1987, quando o termo surgiu, foi proposta uma definição, sendo estabelecido que biomarcadores fossem "indicadores que assinalavam eventos em sistemas ou amostras biológicas sob exposição a contaminantes químicos". A ênfase era o efeito em humanos. Adams (1990) modificou a definição original para incluir respostas de organismos, populações e comunidades aquá- ticas, e Depledge (1994) ampliou a definição, introduzindo respostas comportamentais a definição e outras perspectivas ecotoxicológicas críticas, como diversidade genética.

Os biomarcadores são comumente usados como indicadores bioquímicos, fisiológicos, e histológicos de exposição à xenobióticos ou de efeitos de contaminantes químicos (Huggett et al. 1992). Leonzio \& Fossi (1993) também ampliaram a definição (e consequentemente o uso de biomarcadores) proposta pela National Academy of Sciences, definindo o que propuseram chamar de biomarcadores ecotoxicológicos, como variações bioquímicas, celulares, fisiológicas ou comportamentais que possam ser medidas em amostras de tecidos ou fluidos orgânicos, em organismos ou populações, que possam evidenciar exposição ou efeitos de um ou mais poluentes químicos ou radiações. Mais recentemente, Decaprio (1997) definiu biomarcador como um indicador biológico que evidencia efeito resultante de exposição a um estressor, que pode ser interpretado como evento adaptativo não patogênico ou como séria alteração de um evento funcional, dependendo da toxicocinética e do mecanismo de ação do estressor.

Existem biomarcadores moleculares, celulares e ao nível de indivíduo. As duas características mais importantes dos biomarcadores são: a) permitem identificar as interações que ocorrem entre os contaminantes e os organismos vivos; b) possibilitam a mensuração de efeitos sub-letais. Esta última característica permite pôr em prática ações remediadoras ou, melhor ainda, ações preventivas. Daí a importância e o interesse atual de incorporação da análise de biomarcadores em programas de avaliação da contaminação ambiental.

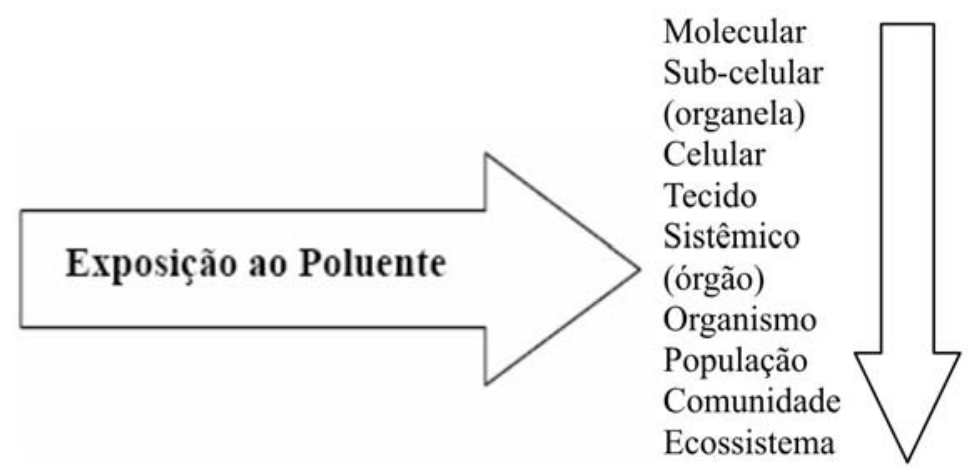

Figura 1. Representação esquemática da ordem seqüencial de respostas a poluentes dentro de um sistema biológico. Figure 1. Schematic representation of the sequence of responses to exposition to pollutants of a biological system. 


\section{CLASSIFICAÇÃO DOS BIOMARCADORES}

Os biomarcadores podem ser classificados como de exposição, efeito ou suscetibilidade.

Os biomarcadores de exposição são alterações biológicas mensuráveis que evidenciam a exposição dos organismos a um poluente. Um exemplo de biomarcadores de exposição são os parâmetros bioquímicos que têm sido testados em peixes com relação a suas respostas a substâncias tóxicas. Entre os indicadores mais investigados nesses animais estão às enzimas presentes no tecido hepático, envolvidas na detoxificação de xenobióticos e seus metabólitos, englobando as enzimas de biotransformação de fase I e fase II e cofatores e as enzimas antioxidantes (Lech \& Vodicnik 1985), como por exemplo, a indução do citocromo P-4501 (CYP1A) monoxidase, sob exposição a hidrocarbonetos poliaromáticos (Bucheli \& Fent 1995). Esses biomarcadores são muito sensíveis e específicos para os compostos referidos. Uma das grandes vantagens do uso desses biomarcadores é o baixo custo, que chega a ser 100 a 200 vezes mais barato em relação ao de análise química (Schlenk 1999).

Os biomarcadores de efeitos, em geral, não são específicos em relação aos estressores e não fornecem informações sobre a sua natureza, mas são característicos da ocorrência de um estresse que poderá ser reversível tão $\operatorname{logo}$ o estressor cesse a atuação. São caracterizados pela indução de mecanismo de defesa celular, que se iniciam sempre como uma resposta adaptativa em nível molecular/ bioquímico. Entretanto, se esse mecanismo falha ou se sua capacidade de resposta é ultrapassada, poderão ser desencadeadas alterações fisiológicas ou histológicas, podendo ser irreversíveis, dependendo da capacidade do sistema ou órgão em responder ao estressor. Assim, o organismo pode ter afetada sua capacidade de reprodução ou crescimento. Alguns desses biomarcadores sáo órgão-específico como enzimas que são lançadas na corrente sanguínea após lesão em tecidos, como várias hepato-amino-ácidos transaminases, indicativas de respostas adaptativas a estressores são as envolvidas com a peroxidação lipídica ou estresse oxidativo (Winzer et al. 2001).

Os biomarcadores de suscetibilidade podem ser definidos como indicadores de processos que causam variações de repostas ao longo do tempo e entre exposição e efeito (Barrett et al. 1997), determinando condições como: indíviduo sadio, compensação do metabolismo, perturbação das funções, alterações morfológicas e morte. Estas condições aumentam a taxa de transição entre esses dois extremos (exposição e efeito). Os organismos, mesmo da mesma espécie, não respondem igualmente a exposição a xenobióticos. Sexo, jejum, estresse pelo confinamento, tamanho e estádio de desenvolvimento são parâmetros devariação nas respostas a estressores, bem como o polimorfismo genético de uma população. Estes biomarcadores correspondem aos chamados biomarcadores de efeito latente (Fossi \& Leonzio 1993), significando que um organismo pode, em determinadas circunstâncias, ter limitada a habilidade de se adaptar ou sobreviver, o que pode ser determinado por mensuração de respostas fisiológicas que, analisadas em conjunto, expressam a diminuição da energia disponível para o crescimento (Nascimento et al. 2006).

Alguns autores (Fossi \& Leonzio 1993) defendem outra classificação que incluiria, além dos citados, os biomarcadores de exposição e efeito, que, além de indicarem a ocorrência de exposição a determinados poluentes, podem ligar, com especificidade, essa exposição ao efeito evidenciando, de modo a caracterizá-lo. A inibição da acetilcolinesterase (AChE) é o melhor exemplo. Essa enzima hidrolisa a acetilcolina, impedindo a ação continuada dessa substância sobre seus receptores em nível das sinapses, propiciando, assim, um controle na transmissão do impulso nervoso. Organofosforados e carbamatos inibem a acetilcolinesterase, causando hiperestimulação dos receptores de acetilcolina, o que impede a contração muscular normal. Por ser específica para esses praguicidas, a inibição dessa enzima é considerada um biomarcador de exposição e de efeito (Nascimento et al. 2006).

O primeiro desafio para o cientista ambiental deve ser a investigação de biomarcadores apropriados para o melhor diagnóstico possível. Para essa escolha deve-se levar em consideração principalmente o contaminante a ser estudado e o melhor modelo biológico.

Os peixes constituem um grupo de grande importância nas avaliações de toxicidade ambiental, pois além de estarem presentes em vários ambientes e apresentarem ampla distribuição geográfica, participam ainda, de diferentes níveis tróficos da cadeia alimentar, sendo considerados como excelentes modelos biológicos de estudo (Rodrigues 2003). 
O mercúrio e seus compostos são ativos quimicamente e, dependendo da concentração atingida no tecido animal, pode desnaturar proteínas e inativar enzimas e alterar a atividade celular tanto pela indução de genes específicos como pela transmissão ou influência de sinais de controle da expressão gênica (Koropatnick \& Zalups 1997). Podem, ainda, modificar as membranas celulares com prejuízo de suas funções, causando a morte celular e a destruição de qualquer tecido com o qual entrem em contato.

Assim, a determinação de parâmetros biológicos selecionados, como histologia, bioquímica e fisiologia, que são conhecidos por variar em resposta aos efeitos tóxicos dos compostos mercuriais, vêm sendo constantemente recomendadas para avaliar o estado de saúde ambiental de ecossistemas aquáticos. Os biomarcadores mais utilizados atualmente para o estudo da contaminação de peixes por mercúrio $(\mathrm{Hg})$ estão descritos a seguir.

\section{PARÂMETROS QUÍMICOS}

A exposição pode ser avaliada por medida da concentração do agente químico em amostras ambientais, como ar (monitorização ambiental), ou através da medida de parâmetros biológicos (monitorização biológica), denominado indicadores biológicos ou biomarcadores. A Monitorização biológica da exposição aos agentes químicos significa a medida da substância ou seus metabólitos em vários meios biológicos, como sangue, urina, ar exalado e outros (por exemplo, mercúrio na urina) (Amorim 2003).

A avaliação biológica da exposição a substâncias químicas só é possível quando estiverem disponíveis suficientes informações toxicológicas referentes aos mecanismos de ação e/ou à toxicocinética dos agentes químicos aos quais os indivíduos estão expostos. Quando a monitorização biológica está baseada na determinação da substância química ou do seu metabólito no meio biológico, torna-se essencial o conhecimento de como a substância é absorvida pelas diferentes vias; posteriormente, de como é distribuída para os diferentes compartimentos do organismo; de como é biotransformada; e, finalmente, de como é eliminada. Além de ser necessário saber também se a substância se acumula ou não no organismo (Bernard \& Lauwerys 1986; Rudiger 1999).

A distribuição de Hg nos órgão dos peixes resulta da ação e interação entre três fatores ecotoxicológicos: (i) condição de exposição, a importância da rota de entrada (água/ingestão da presa) e formas químicas do metal (mercúrio inorgânico, $\mathrm{Hg}$ (II) /MetilHg); (ii) características físico-químicas de biótopos (água e compartimento do sedimento), que afetam a especiação química do mercúrio, bioacumulação e também funções fisiológicas e bioquímicas do organismo; (iii) propriedades estruturais e funcionais de diferentes espécies de peixes que poderia afetar as barreiras biológicas e o mecanismo de excreção/ depuração do peixe (Régine et al. 2006).

Desta forma, torna-se imprescindível o estudo químico das concentrações deste metal nos biótopos e em espécies aquáticas de hábitos alimentares diferenciados, uma vez que o MeHg é um organometálico que além de se bioacumular também se biomagnifica ao longo das cadeias alimentares, ou seja, os organismos do topo de cadeia tem concentrações mais elevadas do que aqueles do início da cadeia (Campbell et al. 2003; Dusek et al. 2005; Hammerschmidt \& Fitzgerald, 2006).

\section{PARÂMETROS GENOTÓXICOS}

Estudos recentes apontam que metais como o mercúrio, ferro, cobre e cromo atuam em ciclo redox, produzindo espécies reativas de oxigênio (EROs) (Stohs \& Bagchi 1995). As EROs e o estresse oxidativo gerado por estas induzem a peroxidação de lipídios, danos ao DNA e alteram a homeostasia de diversos minerais essências. Adicionalmente, o estresse oxidativo afeta diversas rotas metabólicas, incluindo aquelas envolvidas no reparo de danos ao DNA, conhecidos como efeitos genotóxico (Prá et al. 2006).

$\mathrm{O}$ emprego de parâmetros genotóxicos, principalmente em organismos aquáticos como forma de avaliação da qualidade hídrica, permite avaliar o efeito dos poluentes no meio hídrico, bem como alterações de seu potencial tóxico ou genotóxico após interação com o ambiente.

Ao nível celular se podem utilizar, pelo menos, dois tipos de bioindicadores como indicativos de dano ao material genético: o teste do micronúcleo e o ensaio cometa,.

Os micronúcleos são massas de cromatina citoplasmática com o aspecto de pequenos núcleos, constituídos principalmente por fragmentos cromos- 
sômicos ou por cromossomos retardados durante a migração anafásica, que se detectam em células interfásicas mediante técnicas simples de coloração, aparecendo no citoplasma como pequenos fragmentos basófilos. Em células de mamíferos, os micronúcleos são conhecidos há muito tempo, e são chamados de corpúsculos de Howell-Jolly. Os micronúcleos podem aparecer por várias causas, entre elas por falha mitótica, tanto de fragmentos acêntricos de cromossomos, gerados por ruptura (clastogenicidade), quanto de cromossomos completos (aneuploidia), como conseqüência, geralmente, de enfermidades genéticas (Porto et al. 2005).

No Brasil, existem poucos estudos que utilizam o teste de micronúcleos (MNTs) para estabelecer o efeito mutagênico e o efeito clastogênico do mercúrio em peixes. (Nepomuceno et al. 1997, Grisolia \& Cordeiro 2000, Matsumoto \& Cólus 2000) mas nenhum desses estudos avaliou os efeitos genotóxicos na distribuição dos micronúcleos no ambiente ou nos peixes em diferentes níveis tróficos, um fator crítico na avaliação de risco da exposição.

O ensaio cometa é uma técnica capaz de detectar danos de DNA em células individuais. É um teste relativamente novo, mas amplamente aplicado em estudos por ser facilmente manipulável, por ter baixo custo e pelo tempo reduzido para obtenção de resultados. Este ensaio permite a detecção de danos diretos no DNA (Pax-Y-Mino et al. 2002).

Estudos recentes realizados em peixes submetidos a condições experimentais mostraram diferentes conclusões quanto à eficiência do ensaio cometa como biomarcador. Poleza-Lopes (2004), trabalhando com Hoplias malabaricus submetidos à contaminação na água por $\mathrm{MeHg}$, não observou diferença significativa entre o grupo tratado com $\mathrm{MeHg}$ e o grupo controle. Bombail et al. (2001), estudaram eritrócitos de peixes contaminados com diferente concentrações de peróxido de hidrogênio. Neste estudo, o ensaio cometa apresentou diferença significativa entre o grupo controle e os grupos contaminados. Ferraro et al. (2004), aplicaram o ensaio cometa para testar dois contaminantes diferentes, TBT e chumbo, também em H. malabaricus, não encontraram diferença significativa entre o grupo controle e o grupo contaminado em peixes tratados com TBT, porém, nos peixes tratados com chumbo foi encontrada diferença significativa entre os grupos.
As explicações para tais diferenças podem ser várias, desde dosagem do xenobiótico a que o animal foi submetido o que faz com que este não provoque danos diretos ao DNA e as proteínas do sistema de reparo (Ferraro et al. 2004), ou ainda, diferenças na idade e no sexo dos peixes expostos (Polenza-Lopes 2004).

Em trabalhos realizados em áreas consideradas contaminadas, com diferentes animais marinhos e dulcíquolas, foram demonstradas as vantagens do ensaio cometa na avaliação de danos no DNA, porém, estes estudos enfatizam que comparações entre áreas contaminadas não podem ser realizadas facilmente (Lee \& Steinert 2003, Souza \& Fontanetti 2006).

\section{PARÂMETROS HEMATOLÓGICOS}

Os parâmetros do sangue são considerados indicadores fisiohistopatológicos do corpo inteiro e consequentemente é um importante diagnóstico do status estrutural e funcional do peixe exposto à substância tóxica. (Adhikari et al. 2004).

A ausência de estudos sobre a caracterização de células sanguíneas e outros valores hematológicos em diferentes espécies de peixes, provavelmente seja a maior barreira para se usar esta ferramenta na investigação do efeito de contaminantes em peixes (Mela 2004), assim como a escassez de métodos padronizados, diferenças entre sexo e idade, qualidade da água e métodos de captura, podem ser variáveis que dificultam a interpretação dos dados. Por esta razão, é difícil comparar resultados com diferentes espécies e diferentes estudos (Klinger et al. 1996).

O trabalho de Voccia et al. (1994) descreve que a capacidade fagocítica de leucócitos, como macrófagos e granulócitos, presentes no rim-cefálico de Oncorhynchus mykiss foram inibidos após exposição destas espécies de peixes à concentrações sub-letais de $\mathrm{Hg}(0,5 \mathrm{ppb})$, provavelmente devido a citotoxicidade do Hg. Os mesmos autores descrevem que o potencial citotóxico do metilmercúrio para leucócitos da truta foi 10 vezes maior que a do mercúrio inorgânico, atuando nos principais tecidos leucopoiéticos. A ação deste metal parece estar relacionada a interrupção do processo fagocitário em uma de suas etapas: sinalização química, ligação com o alvo, ingestão celular, degranulação, "morte" intracelular e digestão intracelular (Ferreira 2004). 
Mela (2004) observou um aumento nas células no número total de eritrócitos, leucócitos e trombócitos, no grupo de peixes expostos a metilmercúrio em relação ao grupo controle, assim como aumentou o valor do hematócrito e hemoglobina. Alterações na população de leucócitos como reflexo da estimulação ou supressão das defesas naturais (ex. leucócitos fagocíticos) frente à exposição ao $\mathrm{Hg}$, são de importância ecotoxicológica, uma vez que têm o potencial de afetar a susceptibilidade dos indivíduos a doenças. Romão et al. (2006) avaliou os parâmetros sanguíneos de duas espécies de peixes (Geophagus brasiliensis e H. malabaricus) coletados em áreas consideradas poluídas e em áreas de referência. Foram observados índices hematológicos ligeiramente menores em indivíduos coletados na área considerada poluída (hematócrito, hemoglobina e índices de eritrócitos).

Uma linhagem de enzimas específicas do sangue, como as transaminases, podem indicar uma disrupção da membrana celular em certos organismo. Outros parâmetros hematológicos menos específicos como hemoglobina, dosagem de hematócrito, proteínas e glicose, podem ser mais sensíveis a certos tipos de poluentes, ou ainda, níveis de homônios esteróides no sangue ou proteínas normalmente induzidas por esses hormônios podem também indicar certos efeitos reprodutivos devido a disrupção endócrina (Van der Oost et al. 2003).

Low \& Sin (1996) chegaram a descrever um aumento na atividade de liso-enzimas plasmáticas e renais do peixe Trichogaster trichopterus quando submetidos ao Hg. Liso-enzimas são enzimas que compõem a defesa humoral não específica de peixes, e estão presentes no muco, soro, órgãos hematopoéticos e células fagocíticas. Possuem atividade bactericida, variando sua estimulação/produção diretamente em função da situação de estresse do organismo. Sua inibição pode ser perigosa para os peixes uma vez que, por compor a imunidade não específica de inúmeras espécies da ictiofauna, qualquer fator externo (ex. exposição ao $\mathrm{Hg}$ ) que provoque uma diminuição da resistência imunológica frente à patógenos, pode resultar em uma alta taxa de infecção levando inclusive a morte de uma população.

Na Tabela I estão citados alguns trabalhos que utilizaram os parâmetros hematológicos como biomarcador. Ferreira (2004) ao realizar a contagem diferencial de leucócitos de peixes de duas lagoas, uma contaminada e outra considerada como não-impactada, ambas localizadas na Região Norte Fluminense (RJ), concluiu que a lagoa contaminada mostrou uma linfocitopenia e uma trombocitose evidente. Também foi constatado um pequeno aumento no número de granulócitos circundantes no sangue de $H$. malabaricus.

Oliveira- Ribeiro et al. 2006, ao trabalhar com padrão hematológico de $H$. malabaricus expostos sub-cronicamente ao metilmercúrio, não observou diferença significativa entre os eritrócitos dos indivíduos controle e contaminados por $\mathrm{MeHg}$, porém, foi possível notar o aumento de leucócitos, da concentração de hemoglobina, do hematócrito, de neutrófilos e monócitos dos indivíduos contaminados em relação aos indíviduos controle.

\section{PARÂMETROS BIOQUÍMICOS}

Os parâmetros bioquímicos têm sido largamente testados em peixes com relação a suas respostas a substâncias tóxicas. A Tabela II demonstra alguns dos trabalhos científicos que utilizaram diferentes enzimas para avaliar a ação de um contaminante específico em peixes. Entre os indicadores mais investigados

Tabela I. Alguns trabalhos que utilizaram os biomarcadores hematológicos para avaliar a contaminação por mercúrio do peixe, Hoplias malabaricus. Table I. List of papers that employed hematological biomarkers to evaluate contamination by mercury in the fish Hoplias $\underline{\text { malabaricus. }}$

\begin{tabular}{ll}
\hline Referência & Parâmetros hematológicos \\
\hline Mela (2004) & $\begin{array}{l}\text { Contagem de células do sangue, hemoglobinometria, determinação do hematócrito, } \\
\text { índices hematimétricos e morfometria de eritrócitos, no sangue de H. malabaricus } \\
\text { após contaminação in vivo por metilmercúrio. }\end{array}$ \\
Ferreira (2004) & $\begin{array}{l}\text { Contagem diferencial de leucócitos de H. malabaricus para diferenciar o grau de } \\
\text { contaminação de dois ambientes lacustres. }\end{array}$ \\
Oliveira-Ribeiro et al. (2006) & $\begin{array}{l}\text { Estudos de possíveis diferenças hematológicas entre espécies de H. malabaricus } \\
\text { expostos sub-cronicamente ao metilmercúrio. }\end{array}$ \\
\hline
\end{tabular}


Tabela II. Biomarcadores enzimáticos usados atualmente e suas respectivas referências.

Table II. Enzymatic biomarkers currently used to detect contamination in fish with respective references.

\begin{tabular}{lll}
\hline Enzima & Contaminante & Referências \\
\hline ALAD & Chumbo & $\begin{array}{l}\text { Hodson et al. 1977, Goyer \& } \\
\text { Clarkson 2001, Gurer-Orhan et al. 2004 }\end{array}$ \\
EROD & $\begin{array}{l}\text { PAHs e PCDD/Fs, policlorodibenzeno- } \\
\text { para-dioxinas e furanos, e PCBs } \\
\text { (policlorobifenis) }\end{array}$ & $\begin{array}{l}\text { Stegeman \& Hahn 1994, Bucheli \& Fent } \\
1995\end{array}$ \\
& Xenobióticos em geral & \\
GST & Óleo cru e condições de hipoxia & George 1994 \\
LDH & & $\begin{array}{l}\text { Gagnon \& Holdway 1999, Cooper } \text { et al. } \\
\text { 2002 }\end{array}$ \\
\hline
\end{tabular}

nesses animais estão às enzimas presentes no tecido hepático, envolvidas na detoxificação de xenobióticos e seus metabólitos, englobando as enzimas de biotransformação de fase I, fase II, cofatores e as enzimas antioxidantes (Lech \& Vodicnik 1985).

O mercúrio é um metal de alta toxicidade, pois produz no organismo espécies oxigenada altamente reativa como $\mathrm{o}_{2} \mathrm{O}_{2}$ (peróxido de hidrogênio), que induz ao decréscimo dos níveis de glutationa (GSH) e causa a peroxidação lipídica (Stohs \& Bagchi 1995).

A diminuição da glutationa pode reduzir a capacidade celular de destruir os radicais livres e espécies reativas de oxigênio, de modo que aumente o potencial oxidativo da célula. Assim, GSH e suas enzimas associadas exercem um papel protetor dentro da célula. De fato, a conjugação da glutationa com os xenobióticos, seja espontânea, ou catalizada pela glutationa-S-tranferase (GST), diminuem a ação dos xenobióticos e tornam essas moléculas mais solúveis na água, e elas podem ser mais facilmente eliminadas (Boyland \& Chasseaud 1969). Variações nos níveis de glutationa e enzimas envolvidas em atividades associadas à exposição de mercúrio têm sido estudadas, mas as respostas foram variáveis e dependentes das espécies, tempo de exposição, e concentração do metal (Chatterjee \& Bhattacharya 1984, Heisinger \& Scott 1985, Di Simplicio \& Leonzio 1989, Maracine \& Segner 1998, Canesi et al. 1999). Filipak-Neto et al. (2008) submeteram hepatócitos de H. malabaricus a diferentes concentrações de DDT e MeHg, além, da associação entre estes dois contaminantes e concluíram que em todos os tratamentos as atividades das enzimas, catalase (CAT), glicose-6-fosfato desidrogenase (G6PDH), glutationa redutase (GR) e superóxido dismutase (SOD) foram alteradas.
A inibição da atividade da acetilcolinesterase (AChE) tem sido largamente usada como diagnóstico da exposição por compostos anticolinesterases como: organofosforados, carbamatos e pesticidas (Fulton \& Key, 2001). Mais recentemente, estudos indicaram que esse biomarcador é também sensível a outros compostos, incluindo alguns metais e surfactantes (Gill et al. 1990, Labrot et al. 1996, Guilhermino et al. 1998). Rabitto et al. (2005) verificaram inibição da atividade acetilcolinesterase em peixes expostos à chumbo, porém, nesse mesmo estudo peixes foram expostos ao TBT e não apresentaram alterações na atividade desta enzima. Costa et al. (2007) também observaram inibição na atividade da acetilcolinesterase em peixes expostos ao $\mathrm{MeHg}$.

\section{PARÂMETROS HISTOPATOLÓGICOS}

O exame histopatológico é reconhecido cada vez mais como uma ferramenta valiosa para a avaliação do campo do impacto de poluentes ambientais em peixes (Heath 1995, Teh et al. 1997). Lesões específicas que ocorrem nos órgãos de peixes expostos às substâncias tóxicas sob condições de laboratório ajudam a identificar biomarcadores de exposição. Muitos autores estudaram os efeitos histopatológicos do mercúrio nos peixes expostos ao mercúrio inorgânico e metil mercúrio na água em tecidos como fígado, rins, em brânquias e no epitélio olfatório (Filenko et al.1989, Skak \& Baatrup 1993, Handy \& Penrice 1993, Allen 1994; Banerjee \& Bhattacharya 1994, Oliveira Ribeiro et al. 1995, Oliveira Ribeiro et al. 1996, Oliveira Ribeiro \& Torres 1995, Jagoe et al. 1996, Samson \& Shenker 2000, Mela et al. 2007, Oliveira Ribeiro et al. 2008). Estes estudos 
mostraram que as brânquias e o epitélio olfatório estão em contato direto com os poluentes na água, e podem ser usados como indicadores sensíveis da toxicidade relativa do metil mercúrio nos ecossistemas aquáticos devido às interações do mercúrio com os fluxos brânquial dos gases e dos eletrólitos (Heath, 1995) e ocorrências de problemas morfológicos (Jagoe et al. 1996, Oliveira Ribeiro et al. 1996, 2000). Poucos dados estão disponíveis na literatura sobre os aspectos histológicos e ultraestruturais do tecido muscular esquelético de teleósteos frente à contaminação por Hg. Notadamente, a maior parte dos trabalhos publicados refere-se aos aspectos bioquímicos e alterações metabólicas provocadas pela exposição in vitro de culturas de mioblastos ao $\mathrm{Hg}$ ou de experimentos com músculo estriado cardíaco de cobaias (Halbach 1990, Usuki et al. 2001). Por sua vez, Sewry et al. 2002 afirmam que apesar do uso da microscopia eletrônica de transmissão para observação de alterações no músculo esquelético ter diminuindo nos últimos anos devido a aplicações de técnicas de biologia molecular e imunocitoquímica, ainda assim o autor destaca o papel da técnica no diagnóstico de biópsias deste tipo de tecido.

De acordo com Hinton \& Laurén (1990), o fígado é um órgão desintoxicador essencial para o metabolismo e para a excreção de substâncias tóxicas de dentro do corpo. A exposição aos metais pesados pode conseqüentemente causar as mudanças histológicas no fígado e uma investigação histológica de espécimes expostos podem conseqüentemente produzir resultados significativos. Bruslé et al. (1996) afirmam que estudos com histologia de fígados de peixes poderiam servir como modelos para estudar as interações entre fatores ambientais e estruturas hepáticas e suas funções. O efeito prejudicial da poluição do metal pesado na histologia do fígado de peixes pode, entretanto, depender da duração da exposição (crônica ou aguda) e nível de concentração do metal específico.

\section{PARÂMETROS IMUNOLÓGICOS}

Vários pesticidas são conhecidos por afetarem o sistema imune resultando em alterações na resposta humoral e celular. As alterações imunológicas podem resultar em doenças auto-imunes, hipersensibilidades ou alergias, enquanto que a supressão do sistema imune pode levar ao aumento da incidência de doenças infecciosas e até mesmo a transformações neoplásicas (Dean et al., 2001). Sendo assim o uso de biomarcadores imunológicos é muito útil na avaliação do risco toxicológico (Stiller-winkler et al. 1999). Nos mamíferos, foi observado que tanto mediada por célula e humoral (anticorpo - mediada) imunidade pode ser deprimido por poluentes tais como os HPA, PCB, PBB, OCPs (dieldrina, lindano, DDTs e HCB), organometais (. ex. metilmercúrio eorganoestanios) e metais pesados como $\mathrm{Pb}$ e Cd (Von der Oost et al. 2003).

O sistema imune de peixes é bem desenvolvido e pode ser comparado em vários aspectos ao sistema imune de mamíferos (Ellis 1982). Desta forma pode-se supor que a sensibilidade das funções do sistema imune frente à contaminação ambiental é comparável entre diferentes espécies. A função imune natural não específica dos organismos oferece inúmeras possibilidades como fonte de marcadores biológicos na ecotoxicologia.

Vários parâmetros imunológicos podem potencialmente serem utilizados como biomarcadores nos peixes, por exemplo, células brancas sanguíneas (leucócitos), status de linfócitos (contagens das células sanguíneas ou diferenciais) e fatores de defesa não específicas (tais como os níveis de atividade lisossomal aguda e proteínas de fluidos corporais), peso e morfologia de leucócitos produzidos em órgãos, como o baço, timo e o rim, centros de melanomacrofagos (número, tamanho e exame histopatológico) (Mela et al. 2007, Miranda et al. 2008), função macrófago (quimiotaxia, fagocitose, pinocitose e quimioluminescência), um aumento da susceptibilidade a infecções bacteriana, e outros (Weeks et al. 1992, Wester et al. 1994).

Bussolaro et al. (2008) expuseram camundongos a diferentes concentrações de chumbo por $72 \mathrm{~h}$ e concluíram que mesmo em baixas concentrações as funções dos macrófagos eram afetadas através da redução do índice fagocítico, produção de óxido nítrico, estabilidade do sistema endosomal / lisossomal e da adesão celular.

Convém, no entanto, salientar que o sistema imunológico pode ser influenciado por uma grande variedade de estressores, o que implica em que biomarcadores imunológicos podem ser úteis e sensíveis, mas muitas vezes inespecíficos (Weeks et al. 1992). 


\section{PARÂMETROS REPRODUTIVOS E ENDÓCRINOS}

A necessidade de se conhecer os efeitos potenciais dos disreguladores endócrinos tem conduzido a uma demanda por métodos de ensaios in vitro e in vivo para identificar os efeitos biológicos de uma grande variedade de substâncias naturais e sintéticas presentes no meio ambiente.

Recentes estudos experimentais têm investigado a capacidade de baixas doses de metais pesados (cádmio, mercúrio, zinco, chumbo) de causarem alterações nas funções do sistema endócrino. São relatadas alterações no sistema reprodutivo em populações de espécies de peixes, distúrbios na síntese de cortisol em peixes, indução da síntese de VTG (Leblond et al. 1999, Fossi et al. 2004).

Os resultados dos trabalhos existentes que relacionam efeitos reprodutivos e endócrinos causados por Hg e do MeHg na ictiofauna (Who 1990, Wiener et al. 2003) mostram que peixes submetidos a diferentes concentrações de $\mathrm{Hg}$ apresentam alterações hormonais e reprodutivas, e há efeitos adversos sobre o desenvolvimento larval (Who 1990). Verificou-se também que o MeHg pode prejudicar a reprodução dos peixes por afetar o desenvolvimento gonadal e o sucesso de desova dos adultos (Wiener \& Spry 1996), além de reduzir o sucesso de incubação dos ovos e da sobrevivência dos estágios embriolarvais (Mckim et al. 1976, Friedmann et al. 1996, Latif et al. 2001, Hammerchimdt et al. 2002).

Os interferentes endócrinos podem exibir tanto um comportamento estrogênico como androgênico. Estrogênios são esteróides hormonais que regulam e sustentam o desenvolvimento sexual feminino e suas funções reprodutivas. Já os androgênios são esteróides hormonais responsáveis pelo desenvolvimento das características sexuais secundárias masculinas (Hoffman et al. 2003).

A determinação da proteína vitelogenina (VTG) em organismos aquáticos, principalmente peixes, tem sido bastante utilizada na investigação por contaminantes. A vitelogenina é uma fosfolipoglicoproteína sintetizada por todas as fêmeas de ovíparos durante o ciclo reprodutivo; é produzida no fígado e secretada na corrente sanguínea, onde é transportada até os ovários, acumulando-se nos ovócitos em crescimento para ser, então, utilizada como precursora das reservas nutricionais necessárias para o desenvolvimento subse- qüente dos embriões. Em indivíduos imaturos ou em machos, a codificação do gene para esta proteína não existe ou é muito fracamente expressada. Assim, a presença desta proteína no sangue destes organismos representa um biomarcador de exposição, pois sua síntese depende da presença de xenobióticos (Reis Filho et al. 2006).

A síntese de vitelogenina (VTG), é afetado pelo estradiol. Foi demonstrado que peixes expostos a PCBs foram menos capazes de produzir VTG (Spies et al. 1990). O comprometimento da função reprodutora pela diminuição dos níveis plasmáticos de VTG foi reportada para a trutas fêmeas expostas ao Cd (Haux et al. 1988). A síntese de VTG também pode ser induzida em peixes do sexo masculino expostos a substâncias químicas perturbadoras do sistema endócrino, como a alquilfenóis, conduzindo assim a uma chamada feminização dos peixes machos (Gimeno et al. 1996).

O mercúrio (inorgânico ou metilado) causa lesões histopatológicas nas células de Leydig (responsáveis pela síntese de andrógenos) em Clarias sp. expostos cronicamente. Isso veio acompanhado pelo decréscimo dos teores de colesterol plasmático, sugerindo um estímulo do catabolismo hepático ou uma inibição da síntese da molécula. O chumbo foi relatado como supressor da liberação de gonadotropinas em peixes, provavelmente por alterar os níveis de dopamina no hipotálamo, o que inibe a espermatogênese e a produção de andrógenos, bem como reduz o crescimento gonadal e os níveis de estradiol em fêmeas (Heath 1995). Porém, no estudo realizado por Alves Costa (2006) em peixes contaminados por chumbo inorgânico divalente não foi observado nenhum efeito antiestrogênico detectável pelo método empregado (imuno-detecção), após bioensaio (administração via intraperitoneal).

\section{REFERÊNCIAS BIBLIOGRÁFICAS}

ADAMS, S.M. 1990. Status and use of biological indicators for evaluating the effects of stress on fish. American Fisheries Societies Symposium, 8: 1-8.

ADHIKARI, N.P.; PEHG, X.; ALIZADEH, A.; GANTI, S.; NAYAK, S.K. \& KUMAR, S.K. 2004. Multiscale modeling of the surfactant mediated synthesis and supramolecular assembly of cobalt nanodots. Physical Review Letters, 93: 18301- 18304. 
ALLEN, P. 1994. Distribution of mercury in the soft tissues of the blue tilapia Oreochromis aureus (Steindachner) after acute exposure to mercury (II) chloride. Bulletin Environmental Containation Toxicology, 40: 178-184.

ALVES COSTA, J.R.M. 2006. Padronização de Metodologias para o Uso de Biomarcadores de Contaminação Ambiental em Traíra (Hoplias malabaricus, ERYTHRINIDAE): ALA-D, metalotioneína e vitelogenina. Instituo de Biologia. Universidade Federal do Paraná. 120p.

AMORIM, L.C.A. 2003. Os Biomarcadores e sua aplicação na avaliação da exposição aos agentes químicos ambientais. Revista Brasileira de Epidemiologia, 6:158-170.

BANERJEE, S. \& BHATTACHARYA, S. 1994. Histopathology of kidney of Channa punctatus exposed to chronic nonlethal level of elsan, mercury and ammonia. Ecotoxicology Environmental Safety, 29: 265-275.

BARRETT, J.C.; VAINIO, H.; PEAKALL, D. \& GOLDSTEIN, B.D. 1997. $12^{\mathrm{TH}}$ Meeting of ihe scientific group on methodologies for the safety evaluation of chemical: susceptibility to environmental hazards. Environmental Health Perspective, 105: 699-737.

BERNARD, A. \& LAUWERYS, R. 1986. Assessment of human exposure to chemicals through biological monitoring. Pp.17-28. In: Kopfler FC, Craun, GF (eds.), Environmental Epidemiology. Inc., Chelsea: Lewis Publ.

BOMBAIL, V.; AW, D.; GORDON, E. \& BARTTY, J. 2001. Aplication of the comet and micronucleus assays to butterfish (Pholis gunnellus) erythrocyte from the Firth of Forht, cotland, Chemosphere, 44: 383-392.

BOYLAND. E. \& CHASSEAUD, L.F. 1969. The role of glutathione and glutathione S-transferases in mercapturic acid biosynthesis. Advances Enzymology Relatives Areas Molecular Biology, 32: 173-219.

BRUSLÉ, J.; GONZÁLEZ, G. \& ANADON, G. G.1996. The structure and function of fish liver. Pp. 77-93. In: J.S.D. Munshi \& H.M. Dutta, (eds.). Fish Morphology Science Publishers Inc., USA. 300p.

BUCHELI, T.D. \& FENT, K. 1995. Induction of cytochrome P450 as biomarker for environmental contamination in aquatic ecoszstems. Critical Review in Environmental Science Technology, 25: 201-268.

BUSSOLARO, D.; FILIPAK NETO, F.; GARGIONI, R.; FERNANDES, L. C.; RANDI, M. A. F.; PELLETIER, E.; OLIVEIRA RIBEIRO, C. A. 2008. The immune response of peritoneal macrophages due to exposure to inorgani lead in the house mouse Mus musculus. Toxicology in Vitro, 22: 254-260.

CAJARAVILLE, M.P.; BENIANNO, J.M.;BLASCO, J.; PORTE, C.; SARASQUETE, C. \& VIARENGO, A. 2000. The use of biomarkers to assess the impact of pollution in coastal environments of the Iberian Peninsula: a practical approach. The Science of the Total Environment, 247: 295-311.

CAMPBELL. L.M; OSANO, O.; HECKY, R.E. \& DIXON, D.G. 2003. Mercury in fish from three rift valley lakes (Turkana, Naivasha and Baringo) Kenya, East Africa. Environmental Pollution, 125: 281-286.

CANESI, L.; VIARENGO, A.; LEONZIO, C.; FILIPPELLI, M. \& GALLO, G. 1999. Heavy metals and glutathione metabolism in mussel tissues. Aquatic Toxicology, 46: 67-76.

CHATTERJEE, S.\& BHATTACHARYA, S. 1984. Detoxification of industrial pollutants by the glutathione-S-transferase system in the liver of Anabas testudineus (Bloch). Toxicology Letters, 22: 187-198.

COOPER, R.U.; CLOUGH, L.M.; FARWELL, M.A. \& WEST, T.L. 2002. Hypoxiainduced metabolic and antioxidant enzymatic activities in the estuarine fish Leiostomus xanthurus. Journal of Experimental Marine Biology and Ecology, 279: 1-20.

COSTA, J.R.M.A.; MELA, M.; SILVA DE ASSIS, H.C.; PELLETIER, E.; RANDI, M.A.F. \& OLIVEIRA RIBEIRO, C.A. 2007. Enzymatic inhibition and morphological changes in Hoplias malabaricus from dietary exposure to lead (II) or methylmercury. Ecotoxicology and Environmental Safety, 67: 82-88.

DEAN, J.H., HOUSE, R.V., \& Luster, M.I. 2001. Immunotoxicology: Effects of, and Responses to, Drugs and Chemicals. Pp. 1415-1450. In: Taylor \& Francis, (ed), Principles and Methods of Toxicology: Fourth Edition, Philadelphia, Pennsylvania. 1835p.

DECAPRIO, A.P. 1997. Biomarkers: Coming of age for environmental health and risk assessment. Environmental Science Technology, 31: 1837-1848.

DENTON, D.L; STARRETT, G.I; MITH, R.H \& JOHNSON, S.C. 1994. Comparison of hypothesis testing to point estimate techniques for marine toxicity tests. In: Society of Environmental Toxicology and Chemistry (SETAC) Abstracts, $15^{\text {th }}$ annual meeting., Denver- CO, Pensacola FL: SETAC, p. 116-118.

DEPLEDGE, M.H. 1992. Conceptual paradigms in marine ecotoxicology. In: Proceedings of the $12^{\mathrm{TH}}$ Baltic Marine Biologists Symposium. Olsen \& Olsen, Fredensborg, Denmark, pp. 47-52.

DEPLEDGE, M.H. 1994. Genotypic toxicity implications for individuals and populations. Environmental Health Perspectives, 102: 101-104.

DI SIMPLICIO, P. \& LEONZIO, C. 1989. Effects of selenium and mercury on glutathione and glutathione dependent enzymes 
in experimental quail. Bulletin Environmental Contamination Toxicology, 42: 15-21.

DUSEK, L.; SYOBODOVÁ, Z.; JANOUSKOVÁ, D.; VYKUSOVÁ, B.; JARKOVSKÝ, J.; ŠMÍD, R. \& PAVLIS, P. 2005. Bioaccumulation of mercury in muscle tissue of fish in the Elbe River (Czech Republic): multispecies monitoring study 1991-1996. Ecotoxicology and Environmental Safety, 61: 256-267.

ELLIS, A. E. 1982. Differences between the immune mechanisms of fish and higher vertebrates. Pp 5-37. In: R. J. Roberts, (ed.), Microbial diseases of fish. Spec. Publ. Soc. Gen. Microbiol. $129 \mathrm{p}$.

FERRARO, M.V.M.; FENOCCHIO, A.S. ; CESTARI, M.M. ; MANTOVANI, M.S. ; LEMOS, P. M.M. 2004. Genetic Damage induced by trophic doses of lead in the neotropical fish Hoplias malabaricus (Characiformes, Erythrinidae) as revealed by the comet assay and chromosomal aberrations. Genetics and Molecular Biology, 27(2): 270-274.

FERREIRA, A.G. Efeitos ecotoxicológicos da contaminação ambiental por mercúrio em Hopias malabaricus (Traíra BLOCH, 1794 - PISCES - ERYTHRIDAE) de quatro lagoas do Norte do Estado do Rio de Janeiro. Centro de Biociências e Biotecnologia, Campos dos Goytacazes, RJ, UENF. 2004. $165 \mathrm{p}$.

FILENKO, O.F.; XIHUA, D.; XULONG, C. \& YUGI, Z. 1989. Distribution of mercury in the tissues of carp and its biological effects. Hydrobiology, 24: 64-68.

FILIPAK, F.N.; ZANATA, S.M; SILVA de ASSIS, H.C.; NAKAO, L.S.; RANDI, M.A.F. \& OLIVEIRA RIBEIRO, C.A. 2008. Toxic effects of DDT and methylmercury on the hepatocyts from Hoplias malabaricus, Toxicology in Vitro, no prelo.

FOSSI, C. \& LEONZIO, C. 1993. Nondestructive biomarkers in vertebrates. Boca Raton. Floride, Lewis. 313p.

FOSSI, M. C.; CASINI, S.; MARSILI, L.; ANCORA, S.; MORI, G.; NÉRI, G.; ROMEO, T.; AUSILI, A. 2004. Evaluation of ecotoxicological effects of endocrine disrupters during a fouryear survey of the Mediterranean population of swordfish (Xiphias gladius). Marine Environmental Research, 48: 425-429.

FRIEDMMAN, A.S.; WATZIN, M.C.; BRINCK-JOHNSEN, T. \& LEITER, J.C. Low levels of dietary methylmercury inhbit growth and gonadal development in juvenile walleye (Stizostedion vitreum). Aquatic Toxicology, 35: 265-278.

FULTON, M.H. \& KEY, P.B. 2001. Acetylcholinesterase inhibition in estuarine fish and invertebrates as an indicator of organophosphorus insecticide exposure and effects. Environmental Toxicology and Chemistry, 20: 37-45.
GAGNON, M.M. \& HOLDWAY, D.A. 1999. Metabolic enzyme activities in fish gills as biomarkers of exposure to petroleum hydrocarbons. Ecotoxicology and Environmental Safety, 44: 92-99.

GEORGE, S.G. 1994. Enzymology and molecular biology of phase II xenobiotic-conjugating enzymes in fish. Pp. 37-85. In: G.K. Ostrander. (Ed.). Aquatic Toxicology: Molecular, Biochemical and Cellular Perspectives. Lewis Publishers, New York. 560p.

GILL, T.S.; TEWARI, H. \& PANDE, J. 1990. Use of the fish enzime system in monitoring water quality: effects of mercury on tissue enzimes. Comparative biochemistry and physiology, 97: 287-292.

GIMENO, S.; GERRITSEN, A.; BOWMER, T. \& KOMEN, H. 1996. Feminazation of male carp. Nature, 384: 221-222.

GOYER, R.A. \& CLARKSON, T.W. 2001. Toxic effect of metals. Pp. 811- 867. In: C.D. Klaassen, (ed.), Casarett and Doull's toxicology. New York, McGraw-Hill. 1275p.

GRISOLIA, C.K. \& CORDEIRO, C.M.T. 2000. Variability in micronucleus induction with different mutagens applied to several species of fish. Genetic Molecular Biology, 23: 235-239.

GUILHERMINO, L.; BARROS, P.; SILVA, M.C. \& SOARES, A.M.V.M. 1998. Should the use of inhibition of cholinesterases as a specifc biomarker for organophosphate and carbamate pesticides be questioned? Biomarkers, 3: 157-163.

GURER-ORHAN, H.; SABIR, H.U. \& OZGUNES, H. 2004. Correlation between clinical indicators of lead poisoning and oxidative stress parameters in controls and lead-exposed workers. Toxicology, 195: 147-154.

HALBACH, S. 1990. Mercury compounds: lipophilicity and toxic effects on isolated myocardial tissue. Archives Toxicology, 64: 31-5319.

HAMMERSCHIDT, C. R. \& FITZGERALD, W.F. 2006. Bioaccumulation and trophic transfer of methylmercury in Long Island Sound. Environmental Contamination and Toxicology, 51:416-424.

HAMMERSCHIDT, C. R.; SANDHEINRICH, M.B.; WIENER, J.G. \& RADA, R.G. 2002. Effects of dietary MeHg on reproduction of fathed minnows. Environmental Science Technology, 36: 877-883.

HANDY, R.D. \& PENRICE, W.S. 1993. The influence of high oral doses of mercuric chloride on organ toxicant concentrations and histopathology in Rainbow trout, Oncorhynchus mydiss. Comparative Biochemistry and Physiology C, 106: 717-724.

HAUX, C.; BJORNSSON, B.T.; FORLIN, L.; LARSSON, A. \& DEFTOS, L.J. 1988. Influence of cadmium exposure on 
plasma calcium, vitellogenin and calcitonin in vitelogenic rainbow trout. Marine Environmental Research, 24: 199-210.

HEATH, A.C. 1995. Water Pollution and Fish Physiology (Second Edition). Lewis, Boca Raton, FL. 384p.

HEISINGER, J.F. \& SCOTT, L. 1985. Selenium prevents mercuric chloride induced acute osmoregulatory failure without glutathione peroxidase involvement in the black bullhead (Ictalurus melas). Comparative Biochemistry and Physiology C, 80: 295-297.

HINTON, D.E., \& LAURÉN, D.J. 1990. Integrative histopathological effects of environmental stressors on fishes. American Fisheries Society Symposium, 8: 51-66.

HODSON, P.V.; BLUNT, B.R.; SPRY, D.J. \& AUSTENS, K. 1977. Evaluation of erythrocyte-amino levulinic acid dehydratase activity as short-term indicator in fish of a harmful exposure to lead. Journal of the Fisheries Research Board of Canada, 34: 501-508.

HOFFMAN, D.J.; RATTNER, B.A.; BURTON Jr., G. A.; CAIRNS Jr., J.; 2003. Handbook of Ecotoxicology, $2^{\text {nd }}$, (ed.), Lewis Publishers CRC Press LLC: USA, 1290p.

HUGGET, R.J.; UNGER, M.A.; SELIGMAN, P.F. \& VALKIRS, A.O. 1992. The marine biocide tributyltin. Environmental Science \& Technology, 26: 233-410.

JAGOE, D.H.; SHAW-ALLEN, P.L. \& BRUNDAGE, S. 1996. Gill $\mathrm{Na}+, \mathrm{K}+-$ ATPase activity in Largemouth bass Micropterus salmoides from three reservoirs with different levels of mercury contamination. Aquatic Toxicology, 36: 161-176.

KLINGER, R.E.; BLAZER, V.S. \& ECHEVARRIA, C. 1996. Effects of dietary lipid on the hematology of channel catfish (Ictalurus punctatus). Aquaculture, 47: 225-233.

KOROPATNICK, J. \& ZALUPS, R.K. 1997. Effect of non-toxic mercury and cadmium pretreatment on the capacity of human monocytes to undergo lipopolysaccharide-induced activation. British Journal of Pharmacology, 120: 797-806.

LABROT, F. ; RIBERA, D. ; SAINT DENIS, M. \& NARBONNE, J.F. 1996. In vitro and in vivo studies of potential biomarkers of lead and uranium contamination: lipid peroxidation, acetylcholinesterase, catalase and glutathione peroxidase activities in three non-mammalian species. Biomarkers, 1: $21-28$.

LATIF, M.A.; BODALY, R.A.; JOHNSTON, T.A. \& FUDGE, R.J.P. 2001. Effects of environmental and maternally derived methylmercury on the embryonic and larval stages of walley (Stizostedion vitreum). Environmental Pollution, 111: 139-148.

LEBLOND, V. S. \& HONTELA, A. 1999. Effects of in vitro exposures to cadmium, mercury, zinc, and 1-(2-Chlorophenyl)- 1-(4-chlorophenyl)-2,2-dichloroethane on steroidogenesis by dispersed interrenal cells of rainbow trout (Oncorhynchus mykiss). Toxicology and Applied Pharmacology, 157: 16-22.

LECH, J.J. \& VODICNIK, M.J. 1985. Biotransformation. Pp. 526-557. In: G.M. Rand, S.R. Petrocelli. (eds.). Fundamentals of Aquatic Toxicology; Methods and Applications. Hemisphere Publishing Corporation, New York, USA. 666p.

LEE, R. F. \& STEINERT, S.A. 2003. Use of the single cell gel electrophoresis/comet assay for detecting DNA damage in aquatic (marine and freshwater) animals. Mutation Research/ Reviews in Mutation Research, 544: 43-64.

LEONZIO, C. \& FOSSI, M.C. 1993. Nondestructive biomarkers strategy: perspectives and applications. Pp. 297-312. In: M.C. Fossi and C. Leonzio. (eds.). Nondestructive biomarkers in vertebrates. Lewis Publ., London. 368p.

LOW, K.W. \& SIN, Y.M. 1996. In vivo and in vitro effects of mercury chloride and sodium selenite on some non-specific immune responses of Blue Gourami, Trichogaster trichopterus (Pallus). Fish and Shellfish Immunology, 6: 351-362.

MARACINE, M. \& SESNER, H. 1998. Cytotoxicity of metals in isolated fish cells: importance of the cellular glutathione status. Comparative Biochemistry Physiology A, 120: 83-88.

MATSUMOTO, F.E. \& CÓLUS, I.M.S. 2000. Micronucleus frequencies in Astyanax bimaculatus (Characidae) treated with cyclophosphamide and vinblastine sulfate. Genetic Molecular Biology, 23: 489-492.

MCKIM, J.M.; OLSON, G.F.; HOLCOMBE, G.W. \& HUNT, E.P. 1976. Long-term effects of methylmercury chloride on three generation of brook trout (Salvelinus fortinalis): Toxicity, accumulation, distribution and elimination. Journal of the Fisheries Research Board of Canada, 33: 2726-2739.

MELA, M. 2004. Uso de biomarcadores na avaliação dos efeitos do metilmercúrio em Hoplias malabaricus (Block, 1794) (traíra). Dissertação de Mestrado do Instituto de Biologia. Curitiba, PR, UFPR. 123p.

MELA, M.; RANDI, M.A.F; VENTURA, D.F; CARVALHO, C.E.V; PELLETIER, E. \& OLIVEIRA RIBEIRO, C.A. 2007. Effects of dietary methylmercury on liver and kidney histology in the neotropical fish Hoplias malabaricus. Ecotoxicology and Environmental Safety, 68: 426-435.

MIRANDA, A.L.; ROCHE, H.; RANDI, M.A.F.; MENEZES, M.L.; OLIVEIRA RIBEIRO, C. A. 2008. Bioaccumulation of organochlorine pesticides, PCBs, HCB and DDT in tropical freshwater fish species Hoplias malabaricus related to histopathological, physiological and immunological findings. Environment International, 34: 939-949.

NASCIMENTO, I.A.; PEREIRA, S.A. \& LEITE, M.B. 2006. Biomarcadores como instrumentos preventivos de poluição. 
Pp. 413-431. In: P.A Zagatto \& E. Bertoletti. (eds.). Ecotoxicologia Aquática- Princípios e Aplicações. São Paulo. 478p.

NEPOMUCENO, J.C.; FERRARI, I.; SPANO, M.A. \& CENTENO, A.J. 1997. Detection of micronuclei in peripheral erythrocytes of Cyprinus carpio exposed to metallic mercury. Environmetal and Molecular Mutagenesis, 30: 293-297.

OLIVEIRA RIBEIRO, C.A. \& TORRES, R.F. 1995. Acute effects evaluation of inorganic mercury on epidermis of Trichomycterus brasiliensis. Ecotoxicology Environmental Safety, 32: 260-266.

OLIVEIRA RIBEIRO, C.A.; FANTA, E.; TURCATTI, N.M.; CARDOSO, R.I. \& CARVALHO, C.S., 1996. Lethal effects of inorganic mercury on cells and tissues of Trichomycterus brasiliensis. Biocell, 20: 171-178.

OLIVEIRA RIBEIRO, C.A.; FERNANDES, L.N.; CARVALHO, C.S.; CARDOSO, R.I. \& TURCATTI, N.M. 1995. Acute effects of inorganic mercury on olfactory epithelium of Trichomycterus brasiliensis. Ecotoxicology Environmental Safety, 31: 104-109.

OLIVEIRA RIBEIRO, C.A.; FILIPAK, N.F.; MELA, M.; SILVA, P.H.; RANDIB, M.A.F.; RABITTO, I.S.; ALVES COSTA, J.R.M. \& PELLETIER, E. 2006. Hematological findings in neotropical fish Hoplias malabaricus exposed to subchronic and dietary doses of methylmercury, inorganic lead, and tributyltin chloride. Environmental Research,101: 74-80.

OLIVEIRARIBEIRO,C.A.;MESMER-DUDONS;GONZALEZ, P.; YANNICK, D.; BOURDINEAUD, J. ; BOUDOU, A. ; MASSABUAU, J. C. 2008. Chronic and trophic effects of methylmercury on zebrafish muscle fibers. Environmental Toxicology and Pharmacology, 25: 304-305.

OLIVEIRA RIBEIRO, C.A.; PELLETIER, E.; PFEIFFER, W.C. \& ROULEAU, C. 2000. Comparative gill damages and bioaccumulation of inorganic mercury on tropical and nordic fish. Environmental Research A, 83: 286-292.

PAX-Y-MINÕ. C.; DÁVALOS, M.V.; SÁNCHEZ, M.E.; ARÉVALO, M. \& LEONE, P.E. 2002. Should gaps be included in chromosomal aberration analysis? Evidence based on the comet assay. Mutation Research, 516: 57-61.

POLEZA-LOPES, S.C.G. 2004. Avaliação do efeito do metilmercúrio $\left(\mathrm{CH}_{3} \mathrm{Hg}^{+}\right)$em Hoplias malabaricus através da freqüência de aberrações cromossômicas e do ensaios cometa e micronúcleo. Instituto de Biologia. Universidade Federal do Paraná. Curitiba. 54p.

PORTO, J.I.R.; ARAÚJO, C.S.O. \& FELBERG E. 2005. Mutagenic effects of mercury pollution as revealed by micronucleus test on three Amazonian fish species. Environmental Research, 97: 287-292.
PRÁ, D.; GUECHEVA, T.; FRANKE, S.I.R.; KNAKIEVICZ, T.; ERDTMANN, B. \& HENRIQUES, J.A.P. 2006. Toxicidade e Geonotoxicidade do sulfato de cobre em planárias de água doce e camundongos. Journal Brazilian Ecotoxicology, 2: 171-176.

RABITTO, I.S.; COSTA, J.R.M.A.; SILVA DE ASSIS, H.C.; PELLETIER, E.; AKAISHI, F.M.; ANJOS, A.; RANDI, M.A.F. \& OLIVEIRA RIBEIRO, C.A. 2005. Effects of dietary $\mathrm{Pb}$ (II) and TBT (tributytinl) on neotropical fish, Hoplias malabaricus: Histopathological and biochemical findings. Ecotoxicology and Environmental Safety, 60: 147-156.

RÉGINE, M.B, GILLES, D.; YANNICK, D. \& BOUDOU, A. 2006. Mercury distribution in fish organs and food regimes: Significant relationships from twelve species collected in French Guiana (Amazonian basin). Science of the Total Environment, 368: 262-270.

REIS FILHO, R.W.; ARAÚJO, J.C. \& Vieira, E.M. 2006. Hormônio sexuais estrógenos: Contaminante bioativos. Química Nova. 29 (4), 817-822.

ROMÃO, S.; DONATTI, L.; FREITAS, O.M.; TEIXEIRA, J. \& KUSMA, J. 2006. Blood parameter analysis and morphological alterations as biomarkers on the health of Hoplias malabaricus and Geophagus brasiliensis. Brazilian Archives of Biology and Technology, 49: 441-448.

RUDIGER, H.W. 1999. Biomonitoring in occupational medicine. Pp.1027-1039. In: Marquart H, Schäfer SG, McClellan R, Welsch F, (eds.), Toxicology. San Diego: Academic Press. 1330p.

SAMSON, J.C. \& SHENKER, J. 2000. The teratogenic effects of methyl mercury on early development of the zebrafish, Danio rerio. Aquatic Toxicology, 48: 343-354.

SCHLENK, D. 1999. Necessity of defining biomarkers for use in ecological risk assesments. Marine Pollution Bulletin, 39: $48-53$.

SEWRY, C.A.; MULLER, C.; DAVIS, M.; DWYER, J.S.M.; DOVE，J.; EVANS，J.; SCHRODER，R.; FURST，D.; HELLIWELL, T.; LAING, N. \& QUINLIVAN, R.C.M. 2002. The spectrum of pathology in central core disease, Neuromuscular Disorders, 10-12: 930-938.

SKAK, C. \& BAATRUP, E. 1993. Quantitative and histochemical demonstration of mercury deposits in the inner ear of trout, Salmo trutta, exposed to dietary methyl mercury and dissolved mercuric chloride. Aquatic Toxicology, 25: 55-70.

SOUZA, T.S \& FONTANETTI, C.S. 2006. Micronucleus test and observation of nuclear alterations in erythrocytes of Nile tilapia exposed to waters affected by refinery effluent. Mutation Research, Genetic Toxicology and Environmental Mutagenesis, 605: 87-93. 
SPIES, R.B.; STEGEMAN, J.J.; RICE, D.W.Jr.; WOODLIN, B.; THOMAS, P.; HOSE, J.E.; CROSS, J.N. \& PRIETO, M. 1990. Sublethal responses of Platichtus stellatus to organic contamination in San Francisco Bay with emphasis on reproduction. Pp. 87-122. In: McCarthy, J.F., Shugart, L.R. (eds.), Biomarkers of Environmental Contamination. Lewis Publishers, Boca Raton, FL, USA, 471p.

STEGEMAN, J.J. \& HANN, M.E. 1994. Biochemistry and molecular biology of monooxygenase current perspectives on forms, functions and regulation of cytochrome $\mathrm{P} 450$ in aquatic species. Pp. 87-206. In: D.C. Malins \& G.K. Ostrander. (eds.) Aquatic toxicology: Molecular, Biochemical and Cellular Perspectives. Lewis Publishers, Boca Raton, FL. 560p.

STILLER-WINKLER， R.; HADNAGY，W.; LENG， G.; STRAUBE, E.; IDEL, H. 1999. Immunological parameters in humans exposed to pesticides in the agricultural environment. Toxicology Letters, 107: 219-224.

STOHS, S.J. \& BAGCHI, D., 1995. Oxidative mechanisms in the toxicity of metals ions. Free Radical Biology and Medicine, 2: $321-336$.

TEH, S.J.; ADAMS, S.M. \& HINTON, D.E. 1997. Histopatologic biomarkers in feral freshwater fish populations exposed to different types of contaminant stress. Aquatic Toxicology, 37: $51-70$.

USEPA (United States Environmental Protection Agency). 1992. Report on ecological risk assessment guidelines: strategic planning workshop. EPA/630/R-92-002. Washington, DC. 57p. USUKI, F.; YSUTAKE, A.; MATSUMOTO, M. \& HIGUCHI, I. 2001. Chronic low-dose methylmercury administration decreases mitochondrial enzyme activities and induces myopathic changes in rats. Journal of Health Science, 47: $162-167$

VAN DER OOST, R.; BEYER, J. \& VERMEULEN, N.P.E. 2003. Fish bioaccumulation and biomarkers in environmental risk assessement: a review. Environmental Toxicology and Pharmacology, 13: 57-149.

VOCCIA, I.; KRZYSTYNIAK, K.; DUNIER, M.; FLIPO, D. \& FOUNIER, M. 1994. In Vitro Mercury-Related Cytotoxicity and Functional Impairment of the Immune Cells of Rainbow Trout (Oncorhynchus mykiss). Aquatic Toxicology, 29: 37-48.

WEEKS, B.A.; ANDERSON, D.P.; DUFOUR, A.P.; FAIRBROTHER, A.; GOVEN, A.J.; LAHVIS, G.P. \& PETERS, G. 1992. Immunologiocal biomarkers to assess environmental stress, Pp 221-234. In: Huggett, R.J., Kimerly, R.A., Mehrle, P.M., Jr. Bergman, H.L. (eds.), Biomarkers: Biochemical, physiological and Histological Markers of Anthropogenic Stress. Lewis Publishers, Chelsea, MI. USA, 347p.
WESTER, P.W., VETHAAK, D., VAN MUISWINKEL, W.B. 1994. Fish as biomarkers in immunotoxicology. Toxicology, 86: $213-232$.

WHO. Environmental Health Criteria (EHC 101). 1990. Methylmercury. Geneva, 143p.

WIENER，J.G.; KRABBENHOFT， D.P.; HEINZ， G.H. \& SCHEUHAMMER, A.M. 2003. Ecotoxicology of mercury, Pp. 409-463. In: D.J. Hoffman, B.A. Rattner, G.A. Burton, Jr., \& J. Cairns, Jr. (eds.), Handbook of Ecotoxicology, $2^{\text {nd }}$ edition. CRC Press, Boca Raton, Florida, 1312p.

WIENER, J.G. \& SPRY, D.J. 1996. Toxicological significance of mercury in freshwater fish. Pp. 297-339. In: Beyer, W.N.; Heinz, G.H; Redmon-Norwood, A.W. (eds.), Environmental contaminants in Wildlife: Interpreting Tissue Concentration. Florida: Lewis Publishers; Boca Raton, 494p.

WINZER, K.; WINSTON, G.W.; BECKER, W.; VAN NOORDEN, C.J.F. \& KOCHELER, A. 2001. Sex-related responses to oxidative stress in primary cultured hepatocytes of European flounder (Platichthys flesus L.). Aquatic Toxicology, 52: 143-155.

Submetido em 31/07/2008. Aceito em 19/10/2008. 